\title{
CONTROL DE LA SUPERINTENDENCIA NACIONAL DE ADUANAS Y ADMINISTRACIÓN TRIBUTARIA EN OBRAS POR IMPUESTOS, 2019
}

\author{
CONTROL OF THE NATIONAL SUPERINTENDENCY OF CUSTOMS AND TAX \\ ADMINISTRATION IN TAX WORKS, 2019
}

\author{
Carmen Eufemia Ortiz Basauri \\ Universidad Nacional Mayor de San Marcos \\ Lima, Perú \\ ORCID: https://orcid.org/0000-0002-5187-4416 \\ Correo electrónico: orchisr@gmail.com
}

\section{RESUMEN}

Objetivo: Contribuir al fortalecimiento del sistema de control de la administración tributaria SUNAT en los proyectos de inversión pública que se ejecutan en el Perú por el mecanismo de Obras por Impuestos. Método: La investigación fue de tipo cuantitativo, de alcance descriptivo y explicativo, no experimental, de diseño transversal y documental. La muestra estuvo constituida por 50 exfuncionarios de SUNAT a quienes se les aplicó, en noviembre de 2019, una encuesta de 16 ítems con respuesta valorativa de 5 alternativas. Resultados: Los resultados de la investigación muestran que el $80 \%$ de los encuestados están totalmente de acuerdo con la implementación del control preventivo, concurrente y posterior; el $20 \%$ se encuentra de acuerdo, y a $10 \%$ de ellos le es indiferente; ello responde a lo teóricamente esperado. Conclusión: Se concluye que la propuesta planteada explica, en un $80 \%$, que la percepción de los 50 exfuncionarios de SUNAT acerca del fortalecimiento del sistema de control de SUNAT con la implementación del control previo, concurrente y posterior; fortalecerá el mecanismo de Obras por Impuestos.

Palabras clave: Beneficio tributario; certificado de inversión pública; control; Obras por Impuestos; tributación.

\begin{abstract}
Objective: To contribute to the strengthening SUNAT's tax administration control system in public investment projects that are executed in Peru through the mechanism of works for taxes. Method: The research was quantitative, has an explanatory and descriptive range, it is non-experimental, and possesses a cross-sectional and documental design. The sample was made up of 50 former SUNAT officials who, in November 2019, were surveyed on 16 items with an evaluative response of 5 alternatives. Results: The research's results indicate that $80 \%$ of the participants fully agree with the implementation of a preventive, concurrent, and subsequent control; $20 \%$ agree, and $10 \%$ of them are indifferent to the matter; this resonates with the theoretical expectation. Conclusion: The proposal explains, by an average of $80 \%$, that the perception of the 50 former SUNAT officials about the strengthening of the SUNAT's control system with the implementation of a preventive, concurrent, and subsequent control, will strengthen the mechanism of works for taxes.
\end{abstract}

Keywords: Tax benefit; public investment certificate; control; tax works; taxation.

(c) Los autores. Este artículo es publicado por la revista Quipukamayoc de la Facultad de Ciencias Contables, Universidad Nacional Mayor de San Marcos. Este es un artículo de acceso abierto, distribuido bajo los términos de la licencia Creative Commons Atribución 4.0 Internacional (CC BY 4.0) [https://creativecommons.org/licenses/ by/4.0/deed.es] que permite el uso, distribución y reproducción en cualquier medio, siempre que la obra original sea debidamente citada de su fuente original. 


\section{INTRODUCCIÓN}

En los últimos años, la economía peruana ha tenido un proceso de crecimiento sostenido sustentado en las mejoras de los precios de las exportaciones, pero que no se ha visto reflejado en un incremento en los niveles de inversión en infraestructura. Este es un medio importante para que los bienes y servicios generen bienestar y crecimiento social resolviendo así las necesidades en educación, salud, seguridad, y medio ambiente, según la Organización para la Cooperación y el Desarrollo Económicos. Es necesario señalar además, que la infraestructura es un complemento del capital humano que puede aumentar la productividad económica y aprovechar mejor el proceso de crecimiento económico (González, 2018).

En 2010, el Banco de Desarrollo CAF (Corporación Andina de Fomento) en América Latina publicó el libro Infraestructura Pública y Participación Privada, en el cual se anotan conceptos y experiencias en América y España. La publicación resultó muy exitosa para que gobiernos, empresas y bancos privados, conceptualizaran las alianzas público privadas (APP) y su desarrollo en la actualidad (Vassallo, 2015). Al respecto, el Plan Nacional de Infraestructura para la Competitividad del Perú, elaborado por el Ministerio de Economía y Finanzas (MEF), estima que, en el horizonte de los próximos cinco años, la brecha de inversión en infraestructura de acceso básico asciende a 117 183 millones de soles (35 328 millones de dólares americanos) y que, a veinte años, esta brecha ascenderá a 363 452 millones de soles (109 572 millones de dólares americanos) (Ministerio de Economía y Finanzas [MEF], 2020).

Lo descrito guarda relación con lo señalado por la Asociación para el Fomento de la Infraestructura Nacional - AFIN, al indicar que la brecha de infraestructura en el Perú proyectada para el año 2025 ascendería a 160000 millones de soles en las carteras de educación, energía eléctrica, hidráulica, transporte, telecomunicaciones, salud, agua y saneamiento. Incrementar los ingresos fiscales permanentes y perfeccionar la gestión pública son algunos de los lineamientos de política fiscal que señala y destaca el Marco Macroeconómico Multianual 20212024 (MEF, 2020).

La necesidad de desarrollar proyectos de infraestructura en los sectores mencionados difiere con la realidad dada la escasa capacidad del Estado para ejecutar eficientemente lo programado en el Presupuesto Público, debido a problemas como trabas burocráticas o errores en la formulación de los proyectos. Esto se ha consignado en el Reporte de Obras Paralizadas 2019 emitido por la Contraloría General de la República (CGR) en julio de 2018, en el que se presentaban 867 obras públicas paralizadas por un monto contratado de 16871 millones de soles. De estas, solo 12 de ellas corresponden a Asociaciones Público Privadas (APP). Por tal razón, la ejecución de obras con la intervención del sector privado a través de las APP o el Mecanismo Obras por Impuestos es un acierto (CGR, 2019).

La modalidad de Obras por Impuestos (OXI) constituye una interesante alternativa para incentivar la participación del sector privado en la ejecución de proyectos de inversión pública, y una opción eficaz de aplicación de los recursos provenientes del canon. Es por ello, que en 2008 se promulgó la Ley que impulsa la inversión pública regional y local con participación del sector privado (Ley N. $\left.{ }^{\circ} 29230,2008\right)$ y sus modificatorias (Decreto Supremo N. ${ }^{\circ}$ 036-2017-EF, 2017) (Decreto Supremo N. o 294-2018EF, 2018). Desde esa fecha, las inversiones ejecutadas han crecido de 6 millones de soles en sus inicios a 4856,90 millones de soles para diciembre de 2019 (ProInversión, 2020). Según datos del MEF, en el reporte de Transferencias a los Gobiernos Nacional, Local y Regional al 15 de agosto 2019, el monto transferido aumentó en un $40 \%$ del 2016 al 2018, cercano a los tres mil trescientos mil millones de soles (MEF, 2020).

Los trabajos de investigación que han sido ubicados, aun cuando no guardan estrecha relación con el que se está poniendo a consideración, constituyen importantes referencias de interés.

Hidalgo, Narvaez, Sanchez y Zavala (2019) presentaron su tesis Reconstruyendo las Obras por Impuestos: Propuesta de mejora del sistema de obras por impuestos a fin de impulsar la ejecución de proyectos bajo esta modalidad en la universidad ESAN, señalando como objetivo una propuesta para mejorar el sistema actual de Obras por Impuestos y lograr así el incremento de la calidad y el número de obras sujetas a este sistema. Bajo esta perspectiva, el estudio acusa a la identificación y propuestas de mecanismos que coadyuven a la solución de los problemas y limitaciones de la Ley de Obras por Impuestos que ocasionan el incremento de costos directos e indirectos y la ampliación del tiempo de ejecución de la obra, lo que repercute en contra de los intereses del Estado.

Albújar, Santa Cruz, Albújar, Gómez, Quesada y Terrones (2016) presentaron una investigación denominada Obras por Impuestos: factores que promueven la participación de la empresa privada, en la Universidad ESAN. El objetivo general de la investigación fue determinar los factores que influyen en la decisión de las empresas privadas para participar en la modalidad de inversión Obras por Impuestos, sustentando que la principal motivación de las empresas privadas para invertir en Obras por Impuestos es la Responsabilidad Social Empresarial (RSE), ya que se busca mejorar la proyección con la población de la zona de influencia y fortalecer su imagen institucional. 
Harry Chang Yong, exdirector de Promoción de Inversión de Proinversión, señaló que:

El mecanismo de Obras por Impuestos permite a las empresas privadas seleccionar y ejecutar obras de infraestructura pública y constituye una nueva forma de pago del impuesto a la renta que dichas empresas pueden emplear financiando la ejecución de un el proyecto de infraestructura pública de su interés hoy, con cargo al impuesto a la renta futura, hasta por un valor máximo del $50 \%$ de lo pagado en el año fiscal anterior. (Chang, 2015, p. 28).

Para Shack (2016), las Obras por Impuestos son "una modalidad de inversión pública que permite a una empresa o consorcio privado financiar y ejecutar proyectos de infraestructura pública que cuenten con la declaratoria de viabilidad en el marco del Sistema Nacional de Inversión Pública (SNIP)" (p. 35). Por consiguiente, el monto de inversión de las empresas privadas será descontado hasta por la mitad de su respectivo Impuesto a la Renta del anterior año; para ser recuperado a través de la emisión de Certificados de Inversión Pública Regional y Local (CIPRL).

En la búsqueda de información se tuvo acceso también al Proyecto de Ley que fortalece el control gubernamental en los proyectos de inversión ejecutados bajo la modalidad de Obras por Impuestos, que fue presentado por la Contraloría General de la República al Congreso en el mes de febrero de 2019, el cual constituyó un primer acercamiento a la necesidad de implementar mejores mecanismos de control en la ejecución de proyectos de inversión bajo la modalidad de Obras por Impuestos. De la revisión del precitado documento, se ha podido advertir aspectos importantes que denotan que, a esa fecha, las entidades que intervienen en este tipo de proyectos de inversión pública, como es el caso de la Contraloría General de la República (CGR), no cuentan con las herramientas necesarias para aplicar sistemas de control previo, concurrente y posterior que prevean que estos proyectos sean ejecutados con la garantía de constituir una eficaz inversión de los recursos públicos. Asimismo, expone la necesidad de reforzar la participación de la CGR a través de la ejecución de controles concurrentes, que acompañen la ejecución de las obras y no limiten la participación del ente rector del Sistema Nacional de Control a participar en solamente 2 momentos de acuerdo con las normas vigentes.

El proyecto de ley presentado por la CGR no fue aprobado por las instancias competentes en febrero 2019, y es evidente que se continúa limitando la ejecución de controles por parte de dicha entidad, lo cual implica que es necesario afianzar el control a la Superintendencia Nacional de Aduanas y de Administración Tributaria (SUNAT) en sus tres fases: previa, concurrente y posterior; toda vez que tenga directa participación en la ejecución de proyectos de inversión bajo la modalidad de Obras por Impuestos, e intervenga en el desembolso de recursos públicos (CGR, 2019).

Es importante precisar que el mecanismo de Obras por Impuestos permite no solamente financiar la construcción, sino también la rehabilitación y el mantenimiento de una serie de proyectos en beneficio de grandes sectores, bajo la premisa de estar alineados con las necesidades y prioridades de gobiernos regionales o municipales; además, deberán haber sido declaradas como obras de interés, y contar con la declaratoria de viabilidad del Sistema Nacional de Inversión Pública (SNIP).

El sistema de control que se aplica al mecanismo OXI ha merecido el siguiente análisis:

El Ministerio de Economía y Finanzas interviene para otorgar la autorización del financiamiento de la obra pública, previo informe de la CGR y, para la emisión de los certificados de inversión pública, previo informe de la SUNAT.

La Contraloría General de la República, ente rector del Sistema Nacional de Control, solo interviene en dos momentos: emite un informe previo, en el cual únicamente se pronuncia respecto a la capacidad financiera del Estado y emite un informe al Congreso de la República una vez concluidas las obras. En mérito a sus atribuciones y facultades de control, ha emitido diversas normas y procedimientos direccionados principalmente a la verificación de la construcción de la infraestructura y en el caso de las obras de OXI solo actúa por denuncias.

La Superintendencia Nacional de Aduanas y de Administración Tributaria (SUNAT), en el mecanismo de OXI, interviene en dos momentos: comunica al MEF el importe dinerario del $50 \%$ que por concepto de impuesto a la renta corresponde compensar, en mérito a lo que establece la Ley N. ${ }^{\circ} 29230$, a las empresas del sector privado que hubiesen firmado contratos de OXI, y comunica al MEF el importe dinerario que les corresponde otorgar en los certificados de inversión pública CIPRL o CIPGN (ProInversión, 2020).

La afirmación de que el sistema de control para Obras por Impuestos que aplica SUNAT es somero quedó demostrada el 3 octubre de 2019 ante la consulta virtual formulada a dicha institución por la investigadora, mediante la cual se solicitó que se proporcionen datos generales relacionados al control que, como SUNAT, ejercen sobre los beneficios tributarios de impuesto a la renta y al impuesto general a las ventas que se otorga a las empresas que ejecutan obras en el marco del mecanismo de Obras 
por Impuestos. La entidad respondió que, a esa fecha, no se tenía implementado lo solicitado en relación con el beneficio específico vinculado a este mecanismo, y que por ello se encontraban imposibilitados de proporcionar dicha información (SUNAT, comunicación personal, 3 de octubre de 2019).

Resulta pertinente mencionar que reforzar el sistema de control que aplica la SUNAT en la ejecución de esta modalidad de OXI, guarda directa relación con lo establecido en el Marco Macroeconómico Multianual 2021-2024. El documento marco alienta al fortalecimiento de las capacidades de control atribuidas a la administración tributaria. De igual modo, orienta el uso de estrategias que limiten la creación de nuevos regímenes tributarios, así como a la racionalización de los que ya existen. Por último, en lo que respecta al Impuesto a la Renta, se proponen ajustes para fortalecer la base tributaria contribuyendo con la lucha contra la elusión tributaria, y robusteciendo el carácter neutral y equitativo del sistema (MEF, 2020).

El Organismo Supervisor de Contrataciones del Estado (OSCE), según Opinión N. ${ }^{\circ}$ 069-2013/DTN de setiembre de 2013, se pronunció sobre la falta de competencia del Tribunal de Contrataciones del Estado para sancionar a las empresas privadas de OXI, debido a que estas financian con sus propios recursos la ejecución de los proyectos de inversión, y este mecanismo constituye un régimen especial de contratación para las obras que se ejecutan bajo su ámbito (OSCE, 2013).

El Tribunal Fiscal, mediante la Resolución de Observancia Obligatoria N. ${ }^{\circ}$ 08679-3-2019, estableció que un deudor tributario que tenga saldo a favor de impuesto a la renta, si bien no pude efectuar la compensación automática con deudas de otros tributos, ello no constituye una prohibición para que, a su solicitud, la administración tributaria efectúe dicha compensación (MEF, 2019).-

El fortalecimiento del control que debe aplicar la SUNAT en la ejecución de Obras por Impuestos es oportuno y necesario, pues el financiamiento que otorgan las empresas ejecutoras les será retribuido con los certificados de inversión pública que serán utilizados, no solamente como pagos de su impuesto a la renta sino, además, en mérito a la resolución comentada en el párrafo anterior, con deudas de otros tributos.

Por lo antes expuesto, se pretende responder: ¿En qué medida un sistema de control tributario preventivo, concurrente y posterior permitirá a la administración tributaria SUNAT fortalecer el mecanismo de Obras por Impuestos? La respuesta a esta interrogante tiene como objetivo determinar que un sistema de control tributario preventivo, concurrente y posterior, permitiría a la administración tributaria
SUNAT fortalecer el mecanismo de Obras por Impuestos. La hipótesis de esta investigación es: que un sistema de control tributario preventivo, concurrente y posterior permitirá a la SUNAT fortalecer el mecanismo de OXI.

\section{MATERIALES Y MÉTODOS}

Esta investigación fue de tipo descriptiva y la técnica empleada fue el análisis documental. La unidad de estudio estuvo constituida por 50 exfuncionarios de la SUNAT a quienes en noviembre de 2019 se les aplicó una encuesta de 16 ítems con respuesta valorativa de 5 alternativas tipo escala Likert. La información obtenida fue procesada y analizada utilizando un programa R Studio.

\section{RESULTADOS}

En la figura 1, se observa la percepción de los exfuncionarios de la SUNAT acerca del control previo en las empresas que ejecutan y supervisan Obras por Impuestos.

En los seis ítems consultados, casi la totalidad de los encuestados está de acuerdo con lo beneficioso que resulta este mecanismo. El 95\% está de acuerdo con que el control previo evidencia el perfil tributario de las empresas ejecutoras. El 90\% está de acuerdo en que permite verificar el cumplimiento de las obligaciones tributarias de socios y titular. El 94\% señala estar de acuerdo con que el historial de las empresas ejecutoras garantiza que los proyectos se ejecuten conforme a sus objetivos y finalidad. El $96 \%$ está de acuerdo en que coadyuva a la no modificación de los montos contractuales con adendas adicionales. El 91\% está de acuerdo con que permite verificar el que el beneficio tributario se otorgue a empresas que han cumplido con sus obligaciones tributarias. Y, el 95\% está de acuerdo con que el control previo coadyuva al cumplimiento de parámetros de plazo y calidad de la obra a ejecutar.

En la figura 2, se observa la percepción de los exfuncionarios de la SUNAT acerca del control concurrente en las empresas que ejecutan y supervisan Obras por Impuestos.

En los cuatro ítems consultados, casi la totalidad de los encuestados está de acuerdo con lo positivo que constituye este mecanismo. El 94\% está de acuerdo con que el control concurrente permite que se realice la obra conforme a los objetivos y finalidad del proyecto. El 95\% señala estar de acuerdo en que permite que los montos invertidos sean fehacientes. El 100\% está de acuerdo en que permite precisar el importe que debe ser reconocido en el certificado de inversión pública. Y, el 93\% está de acuerdo con que el control concurrente coadyuva a la entrega de la obra en el plazo estipulado y minimiza las contingencias tributarias. 


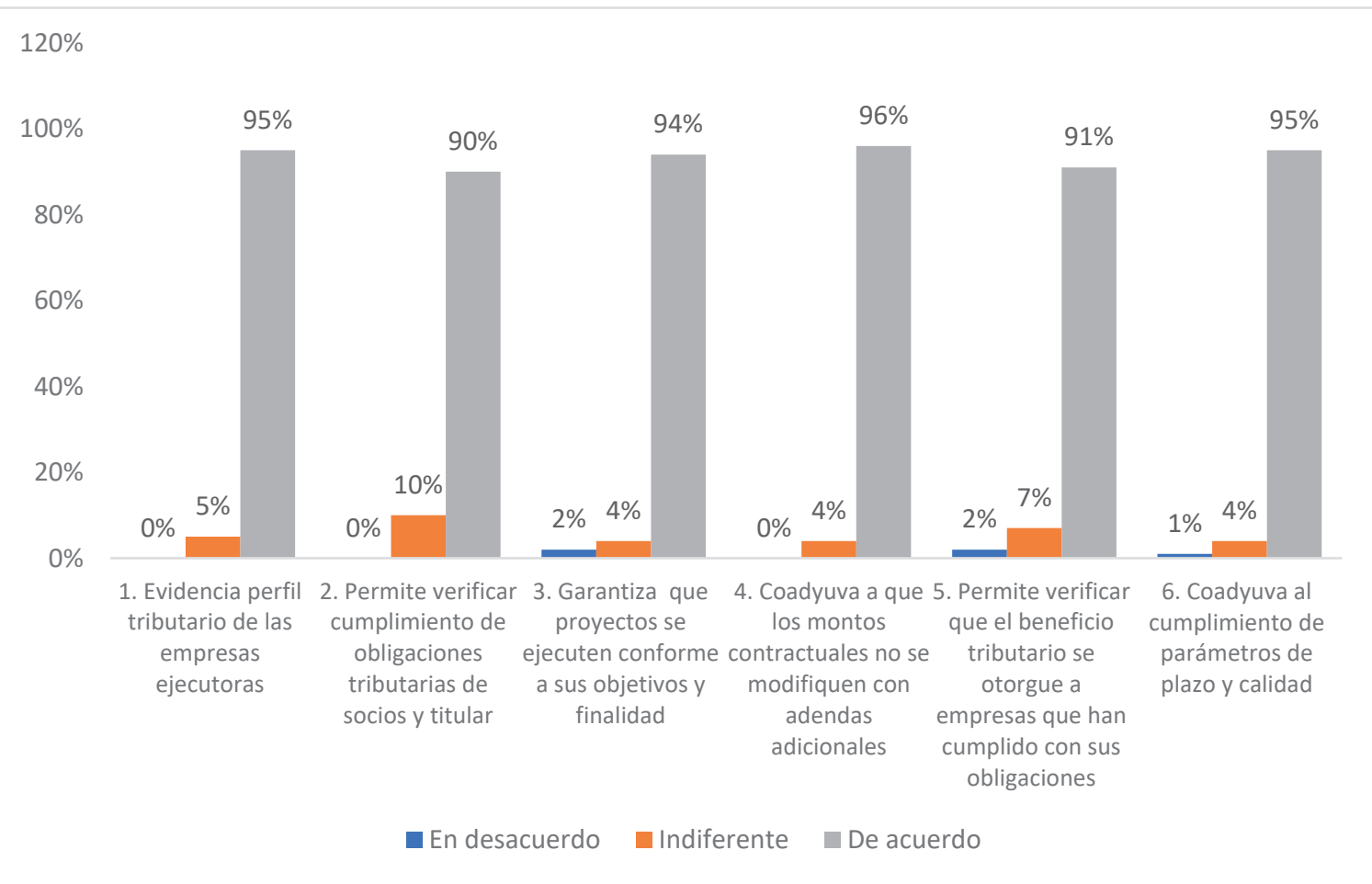

Figura 1. Percepción de exfuncionarios de SUNAT acerca del control previo. Fuente: Elaboración propia

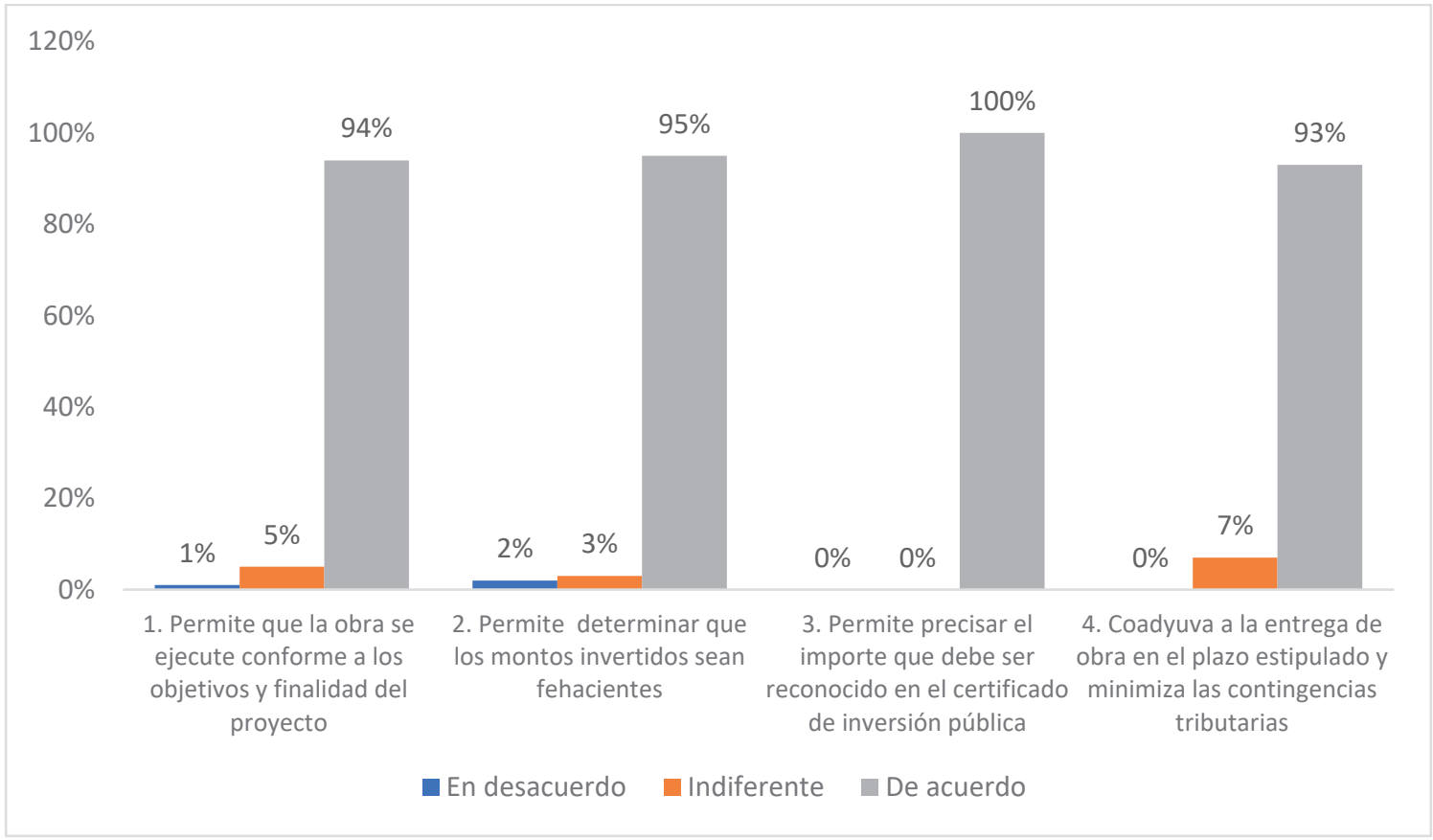

Figura 2. Percepción de exfuncionarios de SUNAT acerca del control concurrente.

Fuente: Elaboración propia 
En la figura 3, se aprecia la percepción de los exfuncionarios de la SUNAT acerca del control posterior en las empresas que ejecutan y supervisan Obras por Impuestos.

En los seis ítems consultados, casi la totalidad de los encuestados está de acuerdo con lo beneficioso que resulta este mecanismo. El 100\% está de acuerdo con que el control concurrente permite determinar el cumplimiento de sus obligaciones tributarias. El $91 \%$ está de acuerdo en que permite que los beneficios tributarios se otorguen con posterioridad al a la verificación del cumplimiento de sus obligaciones tributarias. El 93\% manifiesta estar de acuerdo en que permite determinar el importe del beneficio del impuesto a la renta, a ser reconocido en el certificado de inversión pública. Un 95\% de los exfuncionarios señala estar de acuerdo en que incide en la óptima ejecución dentro del plazo estipulado. El 90\% está de acuerdo en que coadyuva a que el beneficio tributario se vea reflejado en el cumplimiento de las obligaciones tributarias de las empresas ejecutoras. Finalmente, un 92\% expresa estar de acuerdo en que el control posterior permite evitar situaciones irregulares en la emisión de los certificados de inversión pública.

Las facultades de determinación y fiscalización que asisten a la administración tributaria, previstas y tipificadas en el Código Tributario vigente, le permiten verificar la correcta determinación de la obligación tributaria por parte del deudor tributario y modificarla, en caso de omisión o inexactitud; es genérica, para el universo de empresas privadas.

La figura 4 presenta el resultado gráfico de la estructura de los elementos que conforman el circuito de control que debe aplicar para el mecanismo de OXI: (1) el Estado, (2) la empresa beneficiaria, (3) ejecución de la obra, (4) su sistema de control: la empresa supervisora, la CGR y SUNAT, (5) la culminación de la obra y solicitud de Certificado de Inversión Pública (CIP) y (6) la emisión del CIP por el Ministerio de Economía y Finanzas. El fortalecimiento del sistema de control de la administración tributaria SUNAT que este artículo propone, se ubica en 4.

Para el caso de tributos internos como el impuesto a la renta, relacionado al mecanismo de Obras por Impuestos (OXI), se propone la emisión de una resolución por parte de SUNAT que aprobaría el control preventivo, concurrente, y posterior, de las empresas ejecutoras y supervisoras a través de la fiscalización parcial electrónica o verificación; de una manera específica que posibilite:

1. Identificar y cautelar a través del control previo, que el otorgamiento de la buena pro se efectúe a una empresa con adecuado perfil tributario.

2. A través del control concurrente, minimizar las contingencias tributarias que se podrían suscitar, durante la ejecución de una obra.

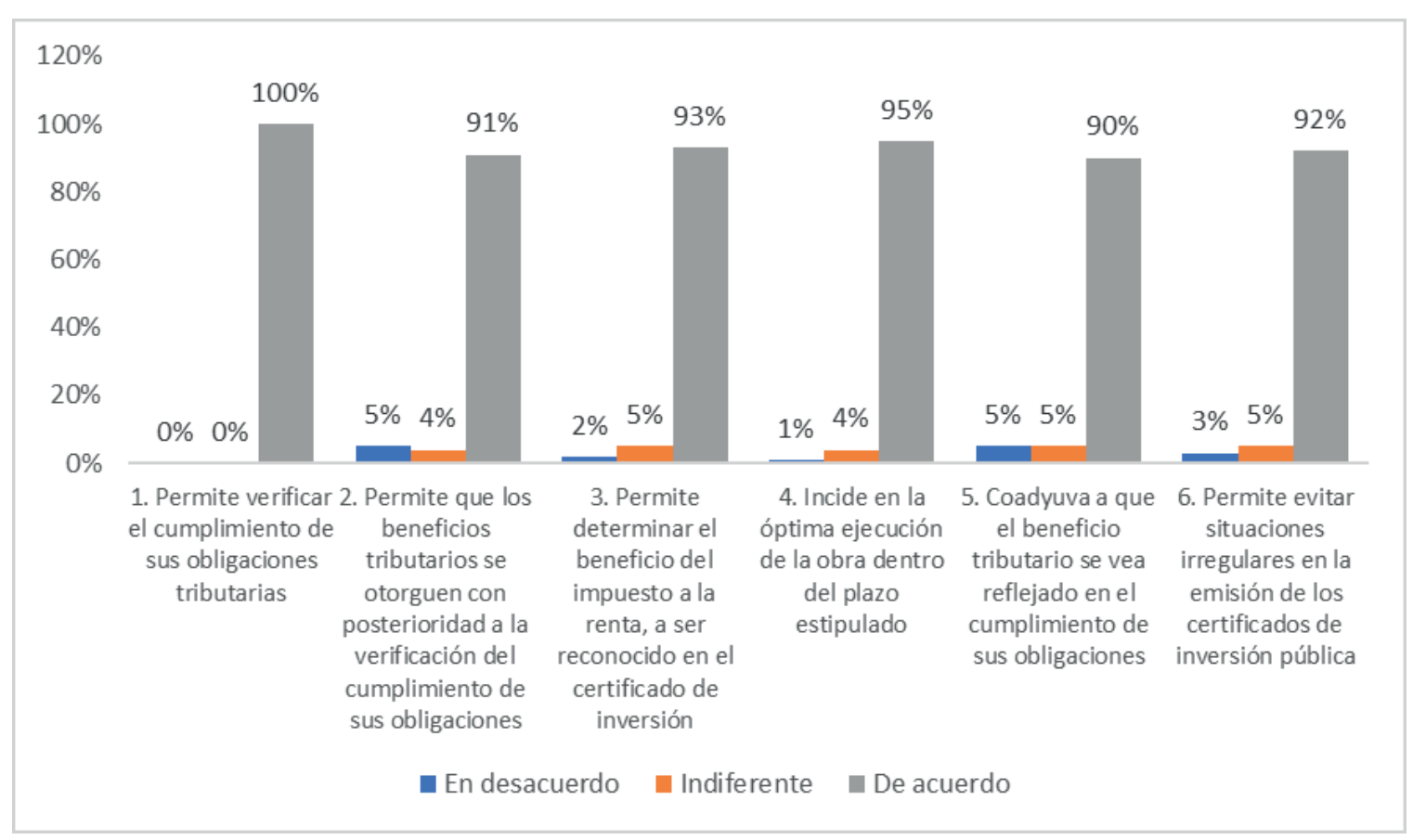

Figura 3. Percepción de exfuncionarios de SUNAT acerca del control posterior.

Fuente: Elaboración propia 


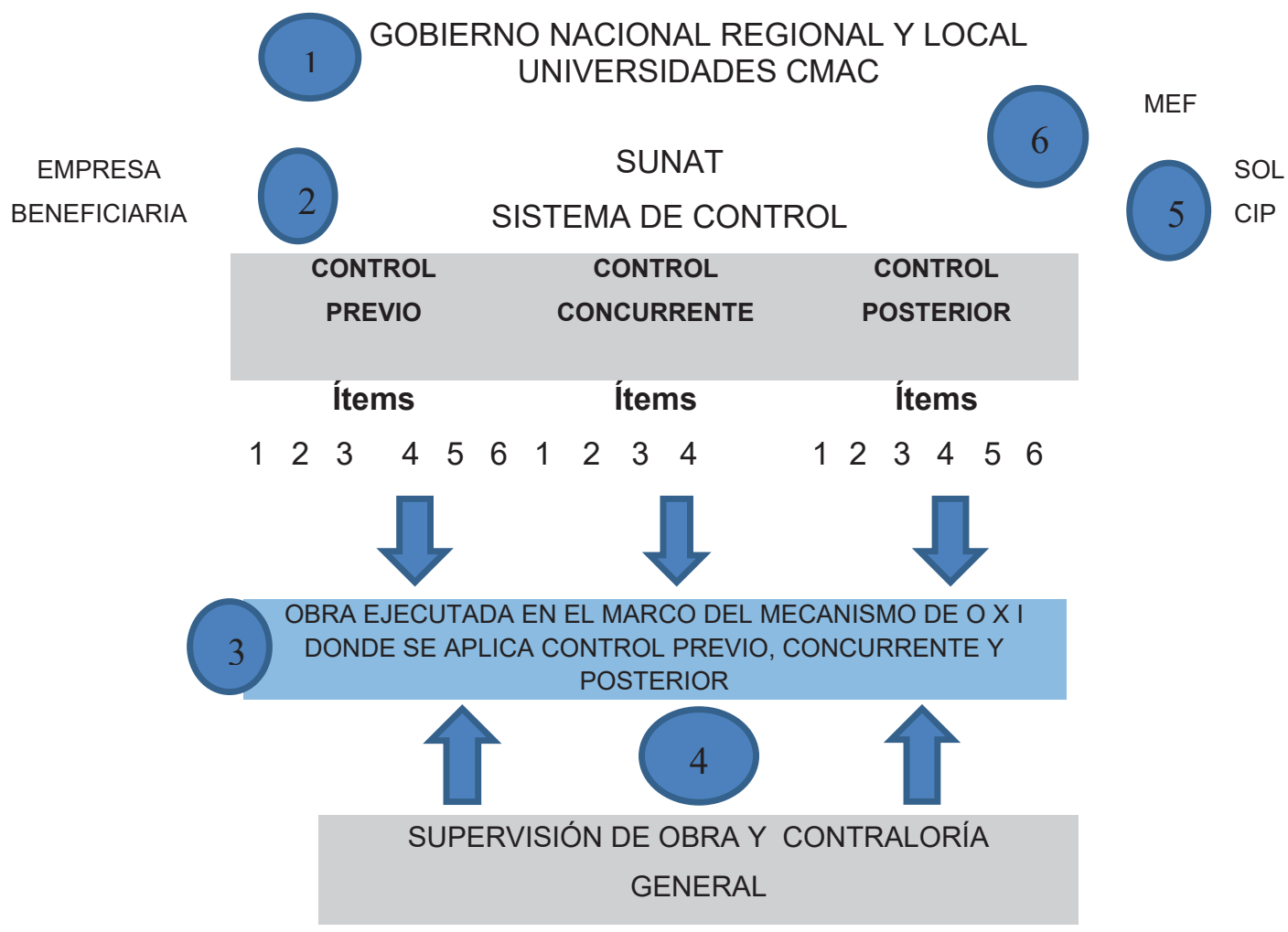

Figura 4. El Sistema de Control de la SUNAT en el Mecanismo de OXI - 2019.

Fuente: Elaboración propia

3. El control posterior permitirá impedir situaciones irregulares en la emisión de los certificados de inversión pública.

Estos tres propósitos constituyen los elementos fundamentales del fortalecimiento del sistema de control de la administración tributaria SUNAT que propone esta investigación.

\section{DISCUSIÓN}

Como el objetivo del artículo es explicar la incidencia del fortalecimiento del sistema de control de la SUNAT en los proyectos de inversión del mecanismo de Obras por Impuestos durante el 2019; los resultados obtenidos muestran la percepción de casi la totalidad de los exfuncionarios de SUNAT acerca de que se aplique el control preventivo, concurrente y posterior. Se evidencia así que, en los tres momentos de control, estos resultados responden a lo teóricamente esperado. Los resultados obtenidos no son comparables a las investigaciones del entorno ya citadas, debido a que estas se enfocan solamente en las bondades y beneficios tributarios que ofrece el mecanismo de OXI para las empresas del sector privado, mas no a su control, lo cual se argumenta en este artículo. Aunque esta situación se puede explicar porque los recursos que financian las obras o servicios provienen del sector privado, no se toma en cuenta que al momento en que se constituyen los pagos a cuenta de impuestos reconocidos en los certificados de inversión pública que emite el Ministerio de Economía y Finanzas, estos pasan a convertirse en obras o servicios públicos financiados con recursos del tesoro público.

Las conclusiones, a las que arriba este artículo, revelan que existe positividad de los mecanismos de control tributario preventivo, concurrente y posterior en la gestión de los recursos tributarios, que le permitiría a la administración tributaria SUNAT fortalecer el mecanismo de Obras por Impuestos. Se ha determinado la factibilidad de aplicar el control preventivo a las empresas ejecutoras y supervisoras de las OXI a fin de establecer su conducta y perfil tributario como garantía de la ejecución eficiente de la obra. Se ha determinado que es beneficioso el control concurrente por parte de la SUNAT, pues permitiría no solamente cautelar en forma oportuna que los montos invertidos en la ejecución de las OXI sean efectuados con idoneidad, razonabilidad, y fehaciencia, sino que, además, minimizaría la determinación posterior de contingencias 
tributarias. Asimismo, se identificó que el control posterior es determinante para que la administración tributaria SUNAT verifique si las empresas ejecutoras y supervisoras cumplieron con sus obligaciones tributarias, cautelando que el beneficio tributario del impuesto a la renta que se les otorgará sea el correcto y se eviten así situaciones irregulares. Se recomienda, por tanto, a la SUNAT: fortalecer el mecanismo de OXI a través de su sistema de control, que permita establecer un sistema de control preventivo, con la finalidad de verificar si las empresas ejecutoras y supervisoras de las Obras por Impuestos evidencien un perfil tributario que garantice la ejecución de la obra; el establecimiento de un sistema de control concurrente, con la finalidad de verificar si los montos invertidos en la ejecución de las obras coinciden con el importe a devolver que será reconocido en el certificado de inversión pública; $y$, finalmente, el establecimiento del sistema de control posterior, a través del cual se pueda verificar si las empresas ejecutoras y supervisoras cumplieron con sus obligaciones tributarias, con la finalidad de constatar el importe correcto del beneficio del impuesto a la renta a ser reconocido en el certificado de inversión pública y evitar situaciones irregulares en su otorgamiento. La implementación de la directiva de cautela previa concurrente y posterior que se ha propuesto a la administración tributaria SUNAT como corolario al trabajo de investigación efectuado, coadyuvará a que la gestión de los recursos tributarios se efectúe correcta y eficientemente.

\section{REFERENCIAS}

Albújar, A., Santa Cruz, E., Albújar, J., Gómez, E., Quezada, K., \& Terrones, S. (2016). Obras por Impuestos: factores que promueven la participación de la empresa privada. Recuperado de: https://www.esan.edu. pe/publicaciones/serie-gerencia-para-el-desarrollo/2016/obras-por-impuestos-factores-que-promueven-la-participacion-de-la-empresa-privada/

Chang, H. (2015). Obras por Impuestos: Poderosa herramienta para la ejecución de obras de infraestructura. Tiempo de Opinión. Recuperado de: https://www.esan. edu.pe/publicaciones/2015/12/03/chang_obras_impuestos.pdf

Contraloría General de la República. (2019). Reporte de obras paralizadas 2019. CGR. Lima. Recuperado de: https://doc.contraloria.gob.pe/estudios-especiales/ documento_trabajo/2019/Reporte_Obras_Paralizadas.pdf

Decreto Supremo N. ${ }^{\circ}$ 036-2017-EF. (2017). Reglamento de la Ley que impulsa la inversión pública regional y local con participación del sector privado. Diario Oficial el Peruano, Lima, Perú.
Decreto Supremo N. ${ }^{\circ}$ 294-2018-EF. (2018). Decreto Supremo que aprueba el Texto Único Ordenado de la Ley $\mathrm{N}^{\circ} 29230$, Ley que impulsa la inversión pública regional y local con participación del sector privado. Diario Oficial el Peruano, Lima, Perú.

González, U. (2018). La política fiscal en Perú en la encrucijada del acceso a la OCDE. Análisis Tributario, 31(362), 11-17.

Hidalgo, S. B., Narvaez, C. D., Sanchez, J. O., \& Zavala, F. (2019). Reconstruyendo las Obras por Impuestos: Propuesta de mejora del sistema de obras por impuestos a fin de impulsar la ejecución de proyectos bajo esta modalidad (Tesis de maestría en Finanzas y Derecho Corporativo). Recuperada de: https://repositorio.esan. edu.pe/bitstream/handle/20.500.12640/1616/2019_ MAFDC_16-2_03_T.pdf?sequence $=1$ \&isAllowed $=y$

Ley N. ${ }^{\circ} 29230$. Ley que impulsa la inversión pública regional y local con participación del sector privado: (28 de febrero de 2017). Diario Oficial el Peruano. Recuperado de: https://busquedas.elperuano.pe/normaslegales/ reglamento-de-la-ley-n-29230-ley-que-impulsa-la-inversion-decreto-supremo-n-036-2017-ef-1491544-2/

Ministerio de Economía y Finazas. (2019). RTF OO 086793-2019. Acuerdos del Tribunal Fiscal. Recuperado de: https://www.mef.gob.pe/contenidos/tribu_fisc/jurisprude/acuer_sala/2019/resolucion/2019_3_08679.pdf.

Ministerio de Economía y Finanzas. (2020). Marco Macroeconómico multianual 2021-2024. Recuperado de: https://www.mef.gob.pe/pol_econ/marco_macro/ MMM_2021_2024.pdf

Organismo Supervisor de Contrataciones del Estado. (2013). Opinión 069-2013. Facultad sancionadora del Tribunal de Contrataciones del Estado. Recuperado de: https://portal.osce.gob.pe/osce/opiniones-2013

ProInversión. (2020). Ranking de Departamentos por Monto de Inversión en Obras por Impuestos 2009 - 2020 (Millones de Soles). Recuperado de: https://www.obrasporimpuestos.pe/0/0/modulos/JER/PlantillaStandard. aspx ?are $=0 \& p r f=0 \& j e r=188 \&$ sec $=0$.

Shack, N. (2016). Reformas de gasto público y crecimiento económico: el caso de las Asociaciones Público-Privadas en el Perú y la inversión "impulsada" ( $\left.\mathrm{N}^{\circ} 171\right)$. Recuperado de: https://repositorio.cepal.org/bitstream/handle/11362/39901/1/S1501197_es.pdf

Vassallo, J. M. (2015). Asociación Público Privada en América Latina. Aprendiendo de la experiencia. Bogotá: CAF. Recuperado de: http://scioteca.caf.com/handle/123456789/758 